\title{
Aleitamento materno no contexto da pandemia de COVID-19: uma revisão de escopo
}

\author{
Breastfeeding in the context of the COVID-19 pandemic: a scoping review \\ Lactancia materna en el contexto de la pandemia COVID-19: una revisión del alcance
}

Eduardo José Braga

ORCID: https://orcid.org/0000-0001-7041-6800

Universidade Federal de São João del Rei, Brasil E-mail: eduardobraga.med@gmail.com

Angelita Gonçalves Meneses

ORCID: https://orcid.org/0000-0002-1579-8524

Universidade Federal de São João del Rei, Brasil

E-mail: angelgoneses@gmail.com

Bárbara Batemarque Sanches Vilela

ORCID: https://orcid.org/0000-0002-3011-2332

Universidade Federal de São João del Rei, Brasil

E-mail: bbatemarque@gmail.com

Ieda Aparecida Diniz

ORCID: https://orcid.org/0000-0002-0032-6401

Universidade Federal de São João del Rei, Brasil

E-mail: ieda.diniz@yahoo.com.br

Flávio Dinz Capanema

ORCID: https://orcid.org/0000-0002-5968-8589

Fundação Hospitalar do Estado de Minas Gerais, Brasil

E-mail: flavio.capanema@ fhemig.mg.gov.br

Gustavo Machado Rocha

ORCID: https://orcid.org/0000-0001-8255-1559

Universidade Federal de São João del Rei, Brasil

E-mail: gusrocha@ufsj.edu.br

Joel Alves Lamounier

ORCID: https://orcid.org/0000-0002-0581-3217

Universidade Federal de São João del Rei, Brasil

E-mail: joel@ufsj.edu.br

Patrícia Peres de Oliveira

ORCID: https://orcid.org/0000-0002-3025-5034 Universidade Federal de São João del Rei, Brasil

E-mail: pperesoliveira@gmail.com

Márcia Christina Caetano Romano

ORCID: https://orcid.org/0000-0002-1819-4689

Universidade Federal de São João del Rei, Brasil

E-mail: marciachristinacs@ufsj.edu.br

\begin{abstract}
Resumo
Introdução: o leite materno é o alimento ideal para a criança, especialmente de forma exclusiva, nos primeiros seis meses de vida e complementado até dois anos de idade ou mais. Na atualidade, um novo desafio se apresenta para a promoção do aleitamento materno: a pandemia de COVID-19. Objetivo: mapear a produção científica que favoreça a promoção do aleitamento materno na pandemia de COVID-19. Métodos: scoping review desenvolvida com base no guia internacional PRISMA-ScR e no método proposto pelo Joanna Briggs Institute. A pesquisa foi realizada em agosto de 2021 nas bases de dados científicas e na literatura cinzenta. Os dados foram coletados, analisados e sintetizados de forma narrativa. Resultados: foram levantadas 2466 publicações, destas, selecionadas 18. As publicações apontam para a baixa possibilidade de transmissão vertical da COVID-19 ou por meio do aleitamento materno e demonstram ainda a incipiente quantidade de evidências científicas acerca do tema. Conclusão: a amamentação, mesmo no contexto da infecção pelo SARS-CoV-2, deve ser promovida e novos estudos são necessários para estabelecer, com significativa evidência científica, a segurança da amamentação no contexto da COVID-19.
\end{abstract}

Palavras-chave: Aleitamento materno; Amamentação; COVID-19; Saúde da criança.

\begin{abstract}
Introduction: breast milk is the ideal food for children, especially exclusively, in the first six months of life and supplemented after up to two years of age or more1. Currently, another new challenge presents itself for the
\end{abstract}


promotion of breastfeeding: the COVID-19 pandemic. Objective: to map the scientific production that favors the promotion of breastfeeding in the COVID-19 pandemic. Methods: scoping review developed based on the international PRISMA-ScR guide and the method proposed by the Joanna Briggs Institute. The survey was conducted in August 2021 in scientific databases and gray literature. Data were collected, analyzed and synthesized in a narrative way. Results: 2466 publications were surveyed, 18 of which were selected. The publications point to the low possibility of vertical transmission of COVID-19 or through breastfeeding and also demonstrate the incipient amount of scientific evidence on the subject. Conclusion: breastfeeding, even in the context of SARS-CoV-2 infection, must be promoted and further studies are needed to establish, with significant scientific evidence, the safety of breastfeeding in the context of COVID-19.

Keywords: Breastfeeding; COVID-19; Child health.

\section{Resumen}

Introducción: la leche materna es el alimento ideal para los niños, especialmente en los primeros seis meses de vida y complementada a partir de los dos años o más. Actualmente, se presenta otro nuevo desafío para la promoción de la lactancia materna: una pandemia de COVID-19. Objetivo: mapear la producción científica que favorece la promoción de la lactancia materna en la pandemia COVID-19. Métodos: análisis de alcance desarrollado en base a la guía internacional PRISMA-ScR y el método propuesto por el Instituto Joanna Briggs. La encuesta se realizó en agosto de 2021 en bases de datos científicas y literatura gris. Los datos se recopilaron, distribuyeron y sintetizaron de forma narrativa. Resultados: Se relevaron 2.466 publicaciones, de las cuales se seleccionaron 18. Las publicaciones apuntan a la baja posibilidad de transmisión vertical del COVID-19 oa través de la lactancia materna y también demuestran la incipiente cantidad de evidencia científica sobre el tema. Conclusión: se debe promover la lactancia materna, incluso en el contexto de la infección por SARS-CoV-2, e introducir nuevos estudios que establezcan, con evidencia científica, la seguridad de la lactancia materna en el contexto de COVID-19.

Palabras clave: Lactancia materna; COVID-19; Salud infantil.

\section{Introdução}

O leite materno é o alimento ideal para a criança, especialmente de forma exclusiva, nos primeiros seis meses de vida e complementado até dois anos de idade ou mais (Ministério da Saúde, 2019). A prática do aleitamento materno oferece aos bebês nutrientes em quantidade e qualidade específicas, dando assim o aporte calórico, proteico, de micronutrientes e vantagens psicológicas, tais como maior vínculo entre mãe e filho, conforto, aconchego, tranquilidade e proteção. Fisiologicamente, favorece o bom desenvolvimento da cavidade oral, estimula a função gástrica, evita deglutição de ar, a superalimentação, confere proteção a doenças infecciosas, principalmente dos tratos respiratório e gastrointestinal bem como a doenças crônicas como obesidade, diabetes mellitus e hipertensão arterial. Para a nutriz, o ato de amamentar oferece proteção contra câncer de mama, ovário e útero, o corpo retorna mais facilmente ao peso pré-gravídico, diminui a incidência de doenças inflamatórias como artrite reumatoide, osteoporose e esclerose múltipla (Carmo \& Portugual, 2020), bem como benefícios do ponto de vista psicológico, melhora do humor e maior vínculo com o bebê (Williams et al., 2020).

Apesar de serem amplamente difundidos os benefícios do aleitamento materno, ainda é um desafio a promoção da amamentação no Brasil. Diversos fatores associam-se ao aleitamento materno e sua duração, como faixa etária e escolaridade da mãe, estado civil, trabalho, apoio do companheiro, amamentação na primeira hora de vida, dentre outros, muitas vezes relacionados com a condição de saúde da mãe e do recém-nascido (Gomes et al., 2020).

$\mathrm{Na}$ atualidade, um novo desafio se apresenta para a promoção do aleitamento materno. A pandemia de COVID-19, iniciada na China e com o primeiro caso confirmado no Brasil, em fevereiro de 2020, provocou incertezas e dúvidas sobre o seu impacto na saúde da criança e, especialmente, sobre a amamentação. Dados brasileiros apontam, no ano de 2021, até a Semana Epidemiológica 26, até três de julho de 2021, dos 1.182.274 casos de SRAG hospitalizados, 10781 (0,9\%) foram em gestantes. Deste total, 7.268 (67,4\%) foram confirmados com a infecção pelo SARS-CoV-2 (Ministério da Saúde, 2020, 2021). A infecção em gestantes e puérperas, além de ter o potencial de maior agravamento, ainda pode causar impacto no cuidado da criança e também no aleitamento materno (Paz et al., 2021), haja visto que devido às incertezas acerca desta nova doença, 
mesmo hospitais certificados, como hospitais amigos da criança, não têm promovido o aleitamento materno no contexto da pandemia de COVID-19 (Gonçalves-Ferri et al., 2021).

A princípio, foi realizada uma revisão narrativa acerca do tema, sendo encontradas duas revisões sistemáticas. Esta revisão de escopo diferiu-se destas duas revisões sistemáticas no tocante à estratégia de busca, às bases de dados utilizadas e à pergunta de pesquisa. Desta forma, tais diferenças somadas à necessidade de reavaliação e síntese da literatura, diante do dinamismo da patologia e volume de produção científica, justificam uma nova revisão para ampliar o conhecimento acerca do tema.

No momento, parece não haver transmissão vertical do SARS-CoV-2. A infecção através do leite materno não foi relatada, embora haja a possibilidade de transmissão aos lactentes pela via respiratória durante a amamentação (Chen et al., 2020; De Rose et al., 2020). Considerando que a pandemia de COVID-19 traz um novo cenário de morbidade, é desafiadora a promoção do aleitamento materno nesse momento atípico. O objetivo deste estudo consiste em mapear a produção do conhecimento que favoreça a promoção do aleitamento materno na pandemia de COVID-19.

\section{Metodologia}

Trata-se de uma revisão de escopo com protocolo de pesquisa aprovado e registrado pelo Open Science Framework (DOI 10.17605/OSF.IO/QT8JY), desenvolvido com base nas recomendações do guia internacional PRISMA-ScR (Tricco et al., 2018) e no método proposto pelo Joanna Briggs Institute, Reviewers Manual 2014 (The Joanna Briggs Institute, 2014), que estabelece cinco etapas: 1) identificação da questão de pesquisa; 2) identificação dos estudos relevantes; 3) seleção dos estudos; 4) análise dos dados; e, 5) agrupamento, síntese e apresentação dos dados (Pereira et al., 2020; The Joanna Briggs Institute, 2014).

A revisão de escopo é utilizada para levantar conhecimentos sobre um determinado tema e sintetizá-lo, seguindo a abordagem sistemática do levantamento das evidências sobre um assunto e identificando os principais conceitos, teorias, fontes e lacunas de conhecimento. Pode ser particularmente indicada para reunir evidências emergentes, pois são adequadas para abordar questões além daquelas relacionadas à eficácia ou experiência de uma intervenção (De Rose et al., 2020; Johns Hopkins, 2021).

Utilizou-se a estratégia Participantes, Conceito e Contexto (PCC) (Tricco et al., 2018) para a construção da pergunta de pesquisa, em que P (participantes) são mães, crianças, recém-nascidos, C (conceito) é aleitamento materno e C (contexto) é a COVID-19. A pergunta de pesquisa estabelecida foi: quais evidências científicas, no contexto da pandemia de COVID-19, estão disponíveis para favorecer a promoção do aleitamento materno?

Os descritores foram obtidos nos Descritores em Ciências da Saúde, sendo: "mães, criança, recém-nascido, aleitamento materno, infecções por coronavírus". Os termos MESH foram obtidos na plataforma US National Library of Medicine National Institutes of Health (PUBMED) através do Medical Subject Headings (MESH), sendo: “mother, infant, newborn infant, breastfeeding e COVID-19”. A estratégia adotada foi a busca pelos estudos utilizando os operadores booleanos: (mães OR mother OR criança OR infant OR recém-nascido OR newborn infant) AND (aleitamento materno OR breastfeeding) AND (infecções por coronavírus OR COVID-19).

A estratégia de busca foi adaptada seguindo as especificidades determinadas por cada base e manteve-se a combinação similar dos descritores. Os critérios de inclusão foram texto completos de pesquisa, publicados na íntegra de dezembro de 2019 até agosto de 2021, nos idiomas inglês, espanhol e português que versam sobre o aleitamento materno seguro em caso de mães infectadas pela COVID-19. Foram excluídos da seleção artigos que não contemplavam a pergunta 
norteadora, editoriais, relatos de experiência, comunicação científica, resenhas, cartas, ensaios teóricos, estudos de caso único e revisões narrativas.

A busca foi realizada no mês agosto de 2021, nas seguintes bases de dados: PUBMED, Literatura Latino-Americana e do Caribe em Ciências da Saúde (LILACS), Medline, através da Biblioteca Virtual de Saúde (BVS), Cummulative Index to Nursing and Allied Health Literature (CINAHL), Web of Science e SCOPUS. A literatura cinzenta também foi pesquisada nas páginas de órgãos oficiais da saúde da criança, como Organização Mundial de Saúde (OMS), Organização Panamericana de Saúde (OPAS), Associação Americana de Pediatria (AAP), Sociedade Brasileira de Pediatria (SBP) e Ministério da Saúde (MS). Ressalta-se que para todas essas bases elencadas, a padronização da busca aconteceu mediante a utilização do Portal de Periódicos da Coordenação de Aperfeiçoamento de Pessoal do Nível Superior (CAPES), por meio da Comunidade Acadêmica Federada ( $\mathrm{CAFe}$ ), com seleção de acesso proveniente de uma instituição federal de ensino superior.

Os títulos e resumos dos artigos encontrados na busca, quando disponíveis, foram lidos e analisados por três pesquisadores independentes (A.G.M., B.B.S.V. e E.J.B). Nas situações de dúvida, um quarto pesquisador (M.C.C.R.) realizou o desempate em plenária. Na etapa seguinte, prosseguiu-se com a leitura na íntegra dos artigos selecionados, visando: a) confirmar a pertinência à pergunta de revisão e, em caso positivo, b) extrair os dados de interesse.

Para a etapa de separação, sumarização e relatório dos elementos essenciais encontrados em cada estudo foi utilizado um formulário estruturado para tabulação desses dados. Esse instrumento permitiu a síntese, interpretação objetiva dos dados e a análise, natureza e distribuição dos estudos incorporados na revisão. Foram agrupados itens como: autores, título, ano de publicação, periódico, país de estudo, objetivos, métodos utilizados, base de dados e síntese dos achados em cada estudo. Assim, em cada publicação foram identificadas e extraídas as informações fundamentais envolvidas no contexto do problema estudado.

Com o intuito de realizar uma avaliação crítica das fontes, optou-se por classificar o nível de evidência e o grau de recomendação dos estudos de acordo com a proposta do Joanna Briggs Institute (The Joanna Briggs Institute, 2013), sendo categorizados de um a cinco e os graus de recomendação em A, B ou C. Os artigos de maior qualidade metodológica são classificados como 1A e os de menor 5C. Pesquisou-se também o fator de impacto dos periódicos em que os estudos foram publicados por meio de consulta à página do Journal Citation Reports (JCR).

\section{Resultados}

A busca realizada originou 2466 publicações inicialmente e todas as etapas de seleção das publicações está ilustrada na Figura 1. Após análise de títulos e resumos, foram pré-selecionados 1039 trabalhos, e, destes 693 foram excluídos por duplicidade. A partir desta etapa, foram selecionadas 346 publicações das quais foram excluídas 282 por não responderem à pergunta do estudo. Das 64 obras restantes, foram selecionadas 18 que apresentavam concordância com os critérios de inclusão, sendo seis na SCOPUS, oito publicadas na PUBMED, duas na BVS e uma na LILACS. Uma publicação incluída pertence à literatura cinzenta, sendo obtida na página da OPAS. 
Figura 1: fluxograma da seleção das publicações nas bases de dados.

\begin{tabular}{|c|c|c|}
\hline \multirow{5}{*}{ 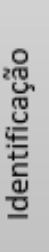 } & \multicolumn{2}{|c|}{ Publicações encontradas nas bases de dados: 2466 publicações } \\
\hline & PUBMED: 892 & LILACS: 32 \\
\hline & SCOPUS: 456 & BVS (Medline): 394 \\
\hline & Web of Science: 494 & CINAHL: 183 \\
\hline & Literatura cinzenta: 15 & \\
\hline \multirow{10}{*}{$\begin{array}{l}E \\
\stackrel{\Xi}{\nu} \\
\text { 은 } \\
\text { 点 }\end{array}$} & \multicolumn{2}{|c|}{$\begin{array}{l}\text { Publicações pré-selecionadas após leitura de títulos e resumos: } 1039 \\
\text { publicações }\end{array}$} \\
\hline & PUBMED:305 & LILACS: 30 \\
\hline & SCOPUS:220 & BVS (Medline): 287 \\
\hline & Web of Science: 6 & CINAHL: 183 \\
\hline & Literatura cinzenta: 8 & \\
\hline & \multicolumn{2}{|c|}{ Após exclusão por duplicação: 346 publicações } \\
\hline & PUBMED: 119 & LILACS: 19 \\
\hline & SCOPUS: 52 & BVS (Medline): 45 \\
\hline & Webof Science: 4 & CINAHL: 100 \\
\hline & Literatura cinzenta: 7 & \\
\hline
\end{tabular}

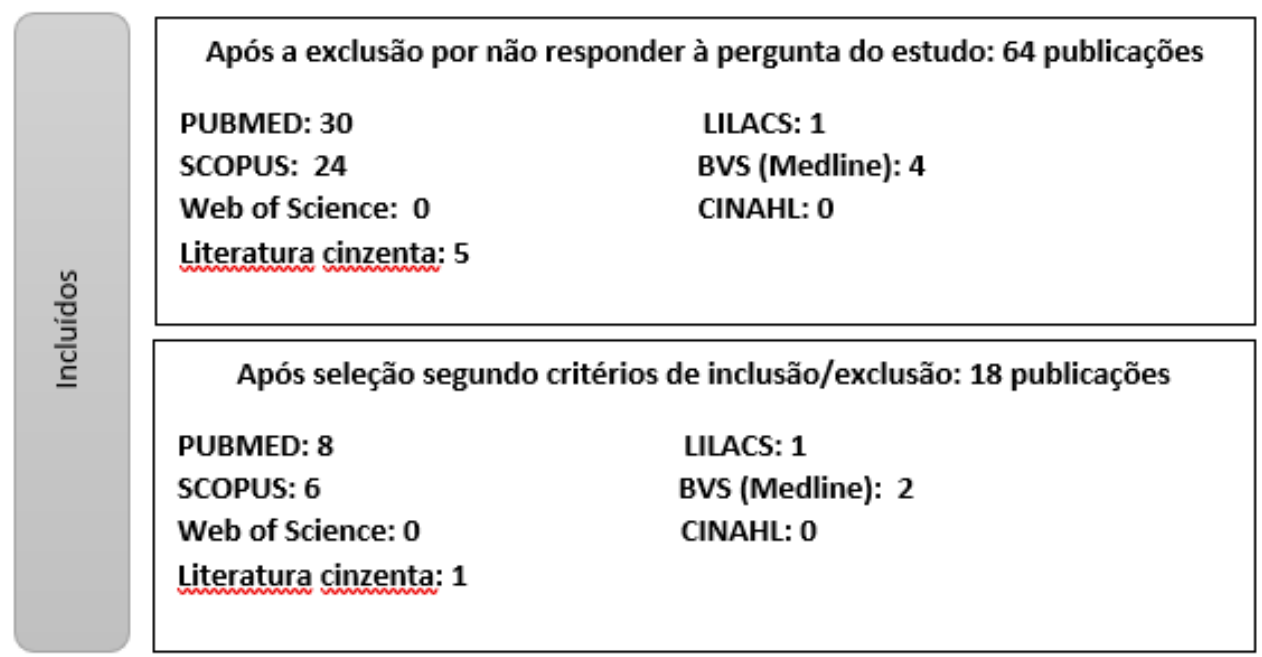

Fonte: adaptado do PRISMA-ScR (Tricco et al., 2018). Divinópolis, Minas Gerais, Brasil, 2021.

Quanto às publicações selecionadas, 50\% tinham amostras da Europa (Bertino et al., 2020; Ceulemans et al., 2020; Davanzo et al., 2020; Dimopoulou et al., 2020; Fernández-Carrasco et al., 2020; Genoni et al., 2020; Pereira et al., 2020; Vassilopoulou et al., 2021; Williams et al., 2020), 27,8\% da Ásia (Ng et al., 2020; Ranganathan et al., 2020; Thanigainathan et al., 2021; Yang et al., 2020) e 22,2\% com participantes dos EUA (Patil et al., 2020; Salvatore et al., 2020), da Austrália (G. J. Walker et al., 2020) e do Brasil (Organização Pan-Americana da Saúde, 2020). A população estudada foi predominantemente composta por gestantes, puérperas e recém-nascidos. De acordo com os desenhos dos estudos, 55,5\% foram revisões (Dimopoulou et al., 2020; Fernández-Carrasco et al., 2020; Genoni et al., 2020; Ng et al., 2020; Vassilopoulou et al., 2021 ; K. Walker et al., 2020; Yang et al., 2020). Os demais 45,5\% divididos em estudos transversais (Bertino et al., 2020; Ceulemans et al., 2020; Patil et al., 2020; Thanigainathan et al., 2021), série de casos (Pereira et al., 2020), coorte (Salvatore et al., 2020), experimental em laboratório (G. J. Walker et al., 2020) e guia de prática clínica (Williams et al., 2020). 
No que se refere à qualificação dos artigos que compuseram essa revisão, o fator de impacto dos periódicos de acordo com JCR variou entre 0,75 e 11,28 sendo que 77,8\% têm impacto menor que cinco. Quanto ao nível de evidência, 38,9\% dos artigos apresentam evidência 5C, 38,9\% nível de evidência B, sendo um 3B, quatro 4B e dois 5B. Duas publicações tiveram classificação 3A, sendo as duas revisões sistemáticas conforme ilustrado no Quadro 1.

Quadro 1: Descrição das publicações conforme tipo de estudo e nível de evidência.

\begin{tabular}{|c|c|c|c|}
\hline Nome do artigo & Tipo de estudo & Síntese dos resultados & $\begin{array}{l}\text { Nível de } \\
\text { evidência }\end{array}$ \\
\hline $\begin{array}{c}\text { Breastfeeding mothers with COVID-19 infection a } \\
\text { case series }\end{array}$ & Série de casos & $\begin{array}{l}\text { Na amostra estudada foi seguro manter AME } \\
\text { mesmo por mães com COVID-19 }\end{array}$ & $4 \mathrm{C}$ \\
\hline $\begin{array}{l}\text { Neonatal management and outcomes during the } \\
\text { COVID-19 pandemic: an observation cohort study }\end{array}$ & Coorte & $\begin{array}{c}\text { Transmissão de COVID-19 é improvável se houver } \\
\text { a higiene correta }\end{array}$ & $3 \mathrm{~B}$ \\
\hline $\begin{array}{l}\text { Coronavirus COVID-19 infection and } \\
\text { breastfeeding: an exploratory review }\end{array}$ & $\begin{array}{c}\text { Revisão } \\
\text { Exploratória }\end{array}$ & $\begin{array}{c}\text { Manter a amamentação seguindo os protocolos de } \\
\text { segurança }\end{array}$ & $5 \mathrm{C}$ \\
\hline $\begin{array}{l}\text { Management and Nutrition of Neonates during the } \\
\text { COVID-19 Pandemic: A Review of the Existing } \\
\text { Guidelines and Recommendations }\end{array}$ & $\begin{array}{l}\text { Revisão } \\
\text { sistemática }\end{array}$ & $\begin{array}{l}\text { Todas as diretrizes recomendam a amamentação ou } \\
\text { alimentação com leite materno ordenhado; a única } \\
\text { exceção são as recomendações chinesas, que } \\
\text { sugeriram evitar a amamentação }\end{array}$ & $5 B$ \\
\hline $\begin{array}{c}\text { SARS-CoV-2 in human milk is inactivated by } \\
\text { pasteurization of the holder, but not by refrigerated } \\
\text { storage }\end{array}$ & $\begin{array}{l}\text { Experimental } \\
\text { em laboratório }\end{array}$ & $\begin{array}{l}\text { A pasteurização inviabiliza o vírus, o congelamento, } \\
\text { apesar de reduzir a carga viral, não. }\end{array}$ & $5 \mathrm{C}$ \\
\hline $\begin{array}{l}\text { Newborns of COVID-19 mothers: short-term } \\
\text { outcomes of colocating and breastfeeding from the } \\
\text { pandemic's epicenter }\end{array}$ & $\begin{array}{l}\text { Estudo } \\
\text { transversal }\end{array}$ & $\begin{array}{c}\text { Na amostra estudada foi seguro manter AME } \\
\text { mesmo por mães com COVID-19, com as devidas } \\
\text { precauções }\end{array}$ & $4 \mathrm{~B}$ \\
\hline $\begin{array}{c}\text { The Importance of Continuing Breastfeeding } \\
\text { during Coronavirus Disease-2019: In Support of } \\
\text { the World Health Organization Statement on } \\
\text { Breastfeeding during the Pandemic }\end{array}$ & $\begin{array}{l}\text { Guia de prática } \\
\text { Clinica }\end{array}$ & $\begin{array}{l}\text { Apoia fortemente a continuação desta prática } \\
\text { durante o COVID-19 }\end{array}$ & $.5 \mathrm{~B}$ \\
\hline $\begin{array}{l}\text { Maternal transmission of SARS-CoV-2 to the } \\
\text { neonate, and possible routes for such transmission: } \\
\text { a systematic review and critical analysis }\end{array}$ & $\begin{array}{l}\text { Revisão } \\
\text { sistemática }\end{array}$ & $\begin{array}{l}\text { Segurança na amamentação em mães com COVID- } \\
19 .\end{array}$ & $5 \mathrm{C}$ \\
\hline $\begin{array}{l}\text { SARS-CoV-2 infections and impact of the } \\
\text { COVID-19 pandemic in pregnancy and } \\
\text { breastfeeding: Results from an observational study } \\
\text { in primary care in Belgium }\end{array}$ & $\begin{array}{l}\text { Transversal e } \\
\text { observacional }\end{array}$ & $\begin{array}{l}\text { Nenhum impacto negativo do bloqueio nas práticas } \\
\text { de amamentação autorreferidas foi observado no } \\
\text { contexto da COVID-19 e metade das mulheres } \\
\text { chegaram a pensar em aumentar a oferta do leite } \\
\text { materno como proteção ao bebê. }\end{array}$ & $4 \mathrm{~B}$ \\
\hline $\begin{array}{l}\text { Breastfeeding of infants born to mothers with } \\
\text { COVID-19: a rapid review }\end{array}$ & $\begin{array}{l}\text { Revisão de } \\
\text { literatura }\end{array}$ & $\begin{array}{c}\text { Todas as intervenções propostas para manter o } \\
\text { aleitamento materno sejam para as mães, bebês e as } \\
\text { famílias, além de salvar vidas, são benéficas, não } \\
\text { exercendo nenhum dano, mesmo com a COVID } \\
\text { materna. }\end{array}$ & $5 \mathrm{C}$ \\
\hline $\begin{array}{c}\text { Aleitamento materno e a doença causada pelo novo } \\
\text { coronavírus (COVID-19) }\end{array}$ & $\begin{array}{c}\text { Revisão } \\
\text { sistemática }\end{array}$ & $\begin{array}{l}\text { Segurança em amamentar mesmo com que a mãe } \\
\text { seja portadora do SARS-CoV-2. }\end{array}$ & $3 \mathrm{~A}$ \\
\hline $\begin{array}{l}\text { Antenatal care, care at birth, and breastfeeding } \\
\text { during the coronavirus (COVID-19) pandemic, } \\
2020\end{array}$ & $\begin{array}{l}\text { Revisão de } \\
\text { literatura }\end{array}$ & $\begin{array}{l}\text { Com base nas evidências disponíveis, as } \\
\text { recomendações da OMS sobre início e continuação } \\
\text { do aleitamento de lactentes e crianças pequenas } \\
\text { também se aplicam às mães com suspeita ou } \\
\text { confirmação de COVID-19. }\end{array}$ & $5 \mathrm{C}$ \\
\hline $\begin{array}{l}\text { Breastfeeding and coronavirus disease-2019: Ad } \\
\text { interim indications of the Italian Society of } \\
\text { Neonatology endorsed by the Union of European } \\
\text { Neonatal \& Perinatal Societies }\end{array}$ & $\begin{array}{l}\text { Revisão de } \\
\text { literatura }\end{array}$ & $\begin{array}{l}\text { A decisão sobre amamentação deve levar em conta } \\
\text { a avaliação cuidadosa dos riscos e benefícios e as } \\
\text { ações baseadas em evidências podem ser úteis para } \\
\text { os profissionais de saúde nestes tempos. }\end{array}$ & $5 \mathrm{C}$ \\
\hline $\begin{array}{c}\text { Breastfeeding in COVID-19: A Pragmatic } \\
\text { Approach }\end{array}$ & $\begin{array}{l}\text { Revisão de } \\
\text { literatura }\end{array}$ & $\begin{array}{l}\text { Segurança em amamentar mesmo com a COVID-19 } \\
\text { materna }\end{array}$ & $5 \mathrm{~B}$ \\
\hline $\begin{array}{l}\text { Breastfeeding and COVID-19: From Nutrition to } \\
\text { Immunity }\end{array}$ & $\begin{array}{c}\text { Revisão } \\
\text { sistemática }\end{array}$ & $\begin{array}{c}\text { Deve-se amamentar desde que respeitadas as } \\
\text { normas sanitárias }\end{array}$ & $3 \mathrm{~A}$ \\
\hline $\begin{array}{c}\text { Is SARS-CoV-2 Transmitted Through } \\
\text { Breastfeeding? }\end{array}$ & $\begin{array}{l}\text { Transversal e } \\
\text { observacional }\end{array}$ & $\begin{array}{c}\text { Os profissionais de saúde que temem os riscos da } \\
\text { transmissão podem levar a práticas restritas de } \\
\text { amamentação. }\end{array}$ & $4 \mathrm{~B}$ \\
\hline
\end{tabular}




\begin{tabular}{|c|c|c|c|}
\hline $\begin{array}{c}\text { Breastfeeding during the novel coronavirus } \\
\text { (COVID-19) pandemic: guidelines and challenges }\end{array}$ & $\begin{array}{c}\text { Revisão de } \\
\text { literatura }\end{array}$ & $\begin{array}{c}\text { A separação entre o recém-nascido e a mãe pode ter } \\
\text { efeitos adversos na relação materno-infantil e pode } \\
\text { prejudicar a amamentação, cuja importância é } \\
\text { indiscutível. }\end{array}$ & 5C \\
\hline $\begin{array}{c}\text { Detection of SARS-CoV-2 in Milk From COVID- } \\
\text { 19 Positive Mothers and Follow-Up of Their } \\
\text { Infants }\end{array}$ & $\begin{array}{c}\text { Transversal e } \\
\text { observacional }\end{array}$ & $\begin{array}{c}\text { Mães positivas não expõem seus recém-nascidos a } \\
\text { um risco adicional de infecção pela amamentação. }\end{array}$ \\
4B \\
\hline
\end{tabular}

Fonte: Autores.

O Quadro 1 enumera de forma sintética os tipos de estudos, as sínteses das evidências bem como níveis de evidência de cada estudo. A maioria das publicações recomendam e confirmam a importância do aleitamento materno, mesmo quando há a infecção da mãe pelo SARS-CoV-2. Uma revisão sistemática incluiu 49 estudos, totalizando 666 neonatos e 655 mães e analisou o tipo de parto, a infecção por COVID-19 e o aleitamento materno. Nesta população, 28 neonatos tiveram infecção pelo SARS-CoV-2, mostrando que a infecção neonatal é relativamente baixa e não se relaciona com o tipo de parto e que a taxa de infecção não é maior quando o bebê nasce de parto normal, é amamentado ou permite o contato com a mãe (K. Walker et al., 2020).

A Sociedade Italiana de Neonatologia conduziu um estudo de revisão no qual avaliou as diretrizes sobre o aleitamento materno e COVID-19. Concluiu que na situação em que uma mãe apresentar a COVID-19 ou estiver sob investigação, o alojamento conjunto é viável e a amamentação é aconselhável com as devidas medidas de controle da infecção. Se a mãe estiver muito doente para cuidar do seu bebê, os autores apontam que este deve ficar separado dela, mas receber seu leite ordenhado, sem necessidade de pasteurização. A justificativa é de que não há evidências apontando que o leite materno é um veículo do SARS-CoV-2 (Davanzo et al., 2020).

Outro estudo de revisão também analisou diretrizes e recomendações sobre o aleitamento materno e COVID-19. As recomendações chinesas e americanas sugerem a separação da mãe e do recém-nascido, enquanto as diretrizes da França, da Itália, Reino Unido, Canadá e OMS sugerem o alojamento conjunto. O Centro para Controle e Prevenção de Doenças dos Estados Unidos orienta avaliar caso a caso. Todas as diretrizes deste estudo recomendam a amamentação ou alimentação com leite materno ordenhado. A única exceção são as recomendações chinesas que contraindicam a amamentação (Genoni et al., 2020).

Um estudo de revisão que avaliou as práticas assistenciais a gestantes e puérperas reforçou que todos os bebês nascidos de grávidas com confirmação ou suspeita de COVID-19 devem ser amamentados. A mesma recomendação quando houver a infecção na puérpera, desde que utilizados os equipamentos de proteção recomendados, com o uso de máscara cirúrgica, realização de higiene das mãos, etiqueta respiratória e desinfecção de rotina de ambientes. Os autores destacam que os benefícios da amamentação superam o risco da transmissão ainda desconhecida do vírus no leite materno e reforçam a importância do acompanhamento psicológico da mãe para favorecer o processo de lactação (Ranganathan et al., 2020).

Uma revisão rápida da literatura sobre a transmissão da COVID-19 de mãe para filho através de leite materno incluiu seis estudos, sendo cinco relatos de caso e uma série de casos com 58 mães. Dessas, 16 apresentaram COVID-19. Nos estudos em que foi testada a presença do SARS-CoV-2 no leite, as amostras foram negativas. Além disso, nenhum recém-nascido foi infectado. Os autores destacam que os benefícios da amamentação podem superar o risco de infecção por SARS-CoV-2 em bebês (Yang et al., 2020).

Uma revisão exploratória sobre os protocolos para assistência da mãe com COVID-19 e seu bebê durante o puerpério foi conduzida. Após analisar 14 documentos, os autores recomendam o aleitamento materno na presença da infecção materna pelo SARS-CoV-2, justificando que as amostras do leite materno analisadas nos estudos continham anticorpos do coronavírus, sendo um fator protetor contra a infecção. Essa revisão também recomenda a doação de leite de mães infectadas para bancos de 
leite e a oferta do leite ordenhado cru para os bebês cujas mães não têm condições físicas de amamentar devido aos sintomas da COVID-19 (Fernández-Carrasco et al., 2020).

Uma revisão acerca das diretrizes sobre o aleitamento materno e a COVID-19 apontou três possibilidades de amamentação. A primeira recomendação refere-se a amamentação direta pela mãe. A segunda proposta é quando a mãe infectada não tem condições para o cuidado ao bebê e este deve receber leite materno ordenhado. E a terceira indicação é o uso de fórmulas. Os autores avaliam que a tomada de decisão sobre o aleitamento materno deve ser feita de forma conjunta entre pais e equipe de saúde (Ng et al., 2020).

Um estudo transversal realizado na atenção primária na Bélgica, em que 2.647 grávidas e 3.823 lactantes participaram, concluiu que 0,3\% das participantes relataram ter testado positivo para SARS-CoV-2. $90 \%$ refutaram que a pandemia afetou suas práticas de amamentação e não indicaram que o coronavírus foi o responsável pela interrupção da amamentação. Além disso, metade das mulheres chegou a pensar em aumentar a oferta de leite materno por causa do coronavírus (Ceulemans et al., 2020).

Uma investigação realizada na Espanha, do tipo relato de séries de casos, acompanhou 22 puérperas com COVID-19 e seus bebês, durante o período médio de 53,5 dias. O estudo objetivou descrever os tipos de aleitamento e dificuldades envolvidas com o manejo da amamentação. Foi relatado que a maior parte dos recém-nascidos $(72,7 \%)$ amamentaram exclusivamente, mas até que o estabelecimento do aleitamento materno exclusivo fosse alcançado, parte desses $(37,5 \%)$ recebeu complemento com fórmula. Durante o acompanhamento, os bebês foram testados para COVID-19 e nenhum deles apresentou resultado positivo ou sintomas da doença. Os autores destacam que, com essa experiência, e, corroborando as recomendações da OMS, é viável a promoção do aleitamento materno em mães com COVID-19, desde que sejam implementadas as medidas de prevenção (Pereira et al., 2020).

Objetivando elucidar as melhores práticas em relação ao controle de infecção no binômio mãe e filho e identificar potenciais fatores de risco associados à transmissão da COVID-19, uma coorte americana avaliou o padrão de amamentação em três hospitais de Nova York, tendo como participantes parturientes. Os recém-nascidos foram acompanhados e testados para COVID-19. De 1.481 partos, 116 (8\%) mães testaram positivo para SARS-CoV-2. Todos os recém-nascidos foram testados com 24 horas de vida e nenhum foi positivo para a infecção. As mães amamentaram seus bebês e foram orientadas a manter os cuidados de prevenção da doença. Os autores sugerem que a transmissão perinatal de COVID-19 é improvável de ocorrer se houver higiene correta e as precauções forem tomadas (Salvatore et al., 2020).

Outra investigação americana, uma pesquisa do tipo transversal, em um hospital de referência para atendimento de gestantes com COVID-19, analisou 118 nascimentos de bebês cujas mães testaram positivo para o SARS-CoV-2. Os autores relatam que apesar da elevada incidência de mães infectadas e essas manterem-se juntas dos bebês, amamentando-os, com os cuidados protocolares, não houve contaminação dos recém-nascidos. Mostraram a importância de boas práticas para a prevenção da doença (Patil et al., 2020).

$\mathrm{Na}$ Austrália foi realizado um experimento em laboratório com o objetivo de analisar a viabilidade viral, por meio da inoculação in vitro do SARS-CoV-2 em amostras de leite humano (LH), após a pasteurização, resfriamento ou congelamento. O estudou encontrou que a pasteurização é um método seguro e não foram encontrados vírus viáveis após o procedimento. No entanto, foram detectadas cargas virais, mesmo que em menor quantidade, nas amostras de LH após resfriamento e congelamento. Tal resultado ratifica a segurança do LH disponibilizado pelos Bancos de Leite Humano (BLH) e traz à reflexão a necessidade de medidas sanitárias rigorosas quanto à não contaminação do LH durante a coleta. Desta forma, o risco de que o SARS-CoV-2 possa ser transmitida a bebês através do leite de doadores é, portanto, amplamente teórico (G. J. Walker et al., 2020). 
A publicação incluída como literatura cinzenta foi produzida pela OPAS que, por meio de uma revisão sistemática, mostrou que a maior parte das amostras de leite de mães foram negativas para o vírus causador da COVID-19. Destacou que em uma investigação foi identificada resposta imune da imunoglobulina secretora A contra o vírus causador da COVID-19. A conclusão do estudo é de que até o momento não há dados suficientes para se inferir sobre a transmissão vertical da COVID19. E que a infecção em bebês é geralmente leve ou assintomática, ao passo que as implicações de não se amamentar podem ser significativas (Organização Pan-Americana da Saúde, 2020).

Um guia de prática clínica europeu foi confeccionado por especialistas com o intuito de informar pediatras sobre as questões acerca da segurança da amamentação em mães com diagnóstico ou suspeita de COVID-19. O documento traz à luz que embora a transmissão vertical materno-fetal não possa ser descartada, ainda não há evidências robustas de que mulheres grávidas infectadas com COVID-19 possam transmitir ao feto o vírus viável. Quanto à transmissão pós-natal, o documento orienta que devido à ausência de estudos relevantes que associem a amamentação ao aumento das taxas de transmissão do SARS-CoV-2 da mãe para o bebê, a Associação Europeia de Pediatria recomenda fortemente a manutenção do aleitamento materno com respeito às medidas de cuidado sanitário vigentes (Williams et al., 2020).

Uma revisão sistemática trouxe que o corpo de conhecimento acumulado sobre o papel do leite materno no desenvolvimento do sistema imunológico neonatal e proteção contra infecções por outros vírus respiratórios é discutido com foco no papel protetor dos anticorpos, microorganismos e vírus fornecidos ao lactente através do leite materno e sua relevância para o caso da SARS-CoV-2. O estudo que avaliou 21 relatos de caso e sete artigos originais, apresenta que a amamentação foi fortemente encorajada quando o bebê e a mãe eram positivos, mas o mesmo não ocorria quando só a mãe era positiva, não havendo evidências de transmissão para o RN. O estudo traz ainda que embora, no momento, esta seja apenas uma suposição para SARS-CoV-2, em outras cepas de SARS-CoV, a lactoferrina mostrou aumentar a imunidade da mucosa e prevenir a ancoragem viral em receptores celulares, citocinas e fatores de crescimento no leite que poderiam promover uma maior ativação imunológica do lactente (Vassilopoulou et al., 2021).

Um estudo transversal indiano foi realizado para verificar a possível transmissão do vírus pela amamentação no contexto nacional com 30 mães positivas para SARS-CoV-2. Destacaram que o RT-PCR dos neonatos também foi realizado às $48 \mathrm{~h}$ e no $5^{\circ}$ dia de vida e todos foram negativos. Todas as amostras de leite materno foram negativas, exceto uma que repetida em 24 horas também foi negativa, não encontrando evidências para a transmissão de SARS-CoV-2 de mãe para filho por meio do leite materno na população estudada (Thanigainathan et al., 2021).

Uma outra revisão, que avaliou as diretrizes disponíveis sobre o tema, apontou que a separação entre o recém-nascido e a mãe pode ter efeitos adversos na relação materno-infantil e pode prejudicar a amamentação, cuja importância é indiscutível. As diretrizes analisadas quase que universalmente apoiam o alojamento conjunto e a amamentação direta de recém nascidos de mulheres com COVID-19 (Dimopoulou et al., 2020).

Um estudo observacional italiano trouxe que com 13 casos de mães positivas para SARS-CoV-2 a análise de amostras de leite foi negativa e em um caso foi positiva. Treze dos 14 recém-nascidos foram amamentados exclusivamente e acompanhados de perto no primeiro mês de vida. Quatro recém-nascidos apresentaram teste positivo para SARS-CoV-2 e todos foram detectados nas primeiras 48 horas de vida, após o início dos sintomas maternos. Além disso, o curso clínico desses quatro bebês, incluindo aquele que recebeu leite materno positivo para SARS-CoV-2, transcorreu sem intercorrências, e todos eles tornaram-se SARS-CoV-2 negativos dentro de seis semanas de vida, concluindo que mães SARS-CoV-2 positivas não expõem seus recém-nascidos a um risco adicional de infecção pela amamentação (Bertino et al., 2020). 


\section{Discussão}

Esta revisão de escopo mapeou a produção do conhecimento sobre o aleitamento materno na pandemia de COVID-19 e os resultados apontaram para o incentivo à amamentação, considerando suas vantagens para a saúde materna e do bebê.

Os benefícios da amamentação, de fato, vêm sendo discutidos desde tempos remotos. Uma importante publicação sobre aleitamento materno (Victora et al., 2016) traz evidências de seus efeitos positivos ao longo da vida, o que é reforçado pela literatura levantada neste estudo. Quanto aos efeitos de curto prazo aos lactentes, estudos demonstram redução da mortalidade em comparação àqueles que receberam qualquer outro tipo de leite que não o materno (Gomes et al., 2020; Rimes et al., 2019). Em relação à morbidade infantil calcula-se que aproximadamente metade de todos os episódios de diarreia e um terço das infecções respiratórias seriam evitados se a prevalência do aleitamento materno exclusivo fosse aumentada para cerca de $90 \%$ (Williams et al., 2020).

Algumas das publicações selecionadas para esta revisão também realçam a importância e os benefícios do aleitamento materno para o lactente, a mãe e a família (Genoni et al., 2020; Patil et al., 2020; Williams et al., 2020). Um documento científico da Sociedade Brasileira de Pediatria (SBP) intitulado como "Amamentação: A base da vida", traz uma síntese de evidências científicas sobre os benefícios do AM, conferindo redução da morbidade por meio da proteção contra doenças infecciosas. Estas infecções são principalmente do trato respiratório e doenças diarreicas, sendo que a amamentação, reduz, inclusive, o número de internações por estas causas. $\mathrm{O}$ documento traz ainda a redução da mortalidade infantil principalmente no primeiro ano de vida e, a longo prazo, diminuição da prevalência de doenças crônicas como diabetes mellitus, obesidade, leucemias bem como da associação positiva com a inteligência (Sociedade Brasileira de Pediatria, 2018). Outros autores ratificam tais benefícios, reforçando os benefícios citados e acrescentam ainda que para o lactente até os seis meses de idade, o leite materno é ideal, atendendo a todas as necessidades nutricionais, psicológicas e imunológicas (Sullivan \& Thompson, 2020).

Outro benefício do aleitamento materno evidenciado na literatura é na formação de microbioma mais equilibrado no trato gastrointestinal do lactente (Nuriel-Ohayon et al., 2019). Este benefício, por meio de imprinting probiótico e prebiótico, é influenciado diretamente pelo tipo de alimentação do recém-nascido, determinando a colonização intestinal. Em comparação com bebês amamentados, os que foram alimentados com fórmula infantil tiveram uma maior variação de espécies patogênicas como uma super colonização por Clostridium dificile (Cabrera-Rubio et al., 2019). O leite materno estimula o desenvolvimento mais equilibrado do microbioma do bebê, principalmente pelo seu alto teor de oligossacarídeos em elevadas concentrações, diferentemente dos outros tipos de tipos de leite, trazendo também uma maior resiliência desta comunidade microbiana (Cabrera-Rubio et al., 2019).

A literatura aponta ainda para a importância da epigenética associada ao leite materno. A regulação da expressão gênica, promovida por constituintes do leite materno, pode exercer influências na resposta metabólica dos recém-nascidos, sendo um objeto atual de estudo (Santiano et al., 2021).

O surgimento de uma nova doença infectocontagiosa, com capacidade pandêmica, como a COVID-19, reforça a necessidade de valorização de todas as medidas com possibilidade de auxiliar em seu controle. Neste contexto, as medidas de mitigação da doença iniciam-se no momento do parto, amamentação, alojamento conjunto, passando por cuidados domiciliares e seguimento ambulatorial na atenção primária (Ministério da Saúde, 2020). Concomitantemente a essas medidas, a promoção do aleitamento materno necessita ser continuada, empoderando a mãe e a família para essa prática, mesmo em um contexto desfavorável, como o da pandemia de COVID-19 (K. Walker et al., 2020)

Quanto aos cuidados necessários à amamentação segura, um artigo internacional recente (Kuehn, 2021) orienta que a mãe lave as mãos antes e depois de tocar o bebê ou objetos relacionados à amamentação, evite usar bomba para retirada de 
leite compartilhada com outras pessoas e siga rigorosamente as instruções de higiene dos fabricantes, use máscara ou cobertura facial durante a amamentação e/ou manipulação do leite materno, amamente se tiver condições clínicas que a permitam e ter sempre ajuda de cuidadores sem sintomas de COVID-19. O autor traz ainda que mães com risco de exposição laboral que amamentem devem conversar com superiores com o intuito de fazer ajustes no trabalho para reduzir sua exposição ao vírus, como possibilidade de banho e troca de vestimentas utilizadas no trabalho (Sullivan \& Thompson, 2020). Desta forma, após a alta hospitalar, a atenção primaria desempenha um papel primordial no estabelecimento seguro da amamentação, como orientado no documento do MS (Ministério da Saúde, 2020), apesar de estudo que aborda as vulnerabilidades à saúde da criança destacar que o acesso aos serviços de saúde tem sido limitado em tempos da atual pandemia (Cabral et al., 2021).

No tocante às evidências que contraindicaram a amamentação no contexto da COVID-19 materna, das 18 publicações selecionadas neste estudo, apenas uma recomendou a separação entre a mãe e o bebê (Ng et al., 2020). O referido estudo é um consenso escrito por especialistas chineses publicado em junho de 2020, ainda na fase inicial da pandemia, quando o conhecimento sobre todos os aspectos desta nova doença ainda era incipiente, com fracas evidências para contraindicar o aleitamento materno no contexto de COVID-19 materna.

A falta de diretrizes e padronização do aleitamento materno no contexto da COVID-19 inclusive no âmbito hospitalar mostra-se também presente. Um estudo transversal multicêntrico (Gonçalves-Ferri et al., 2021) brasileiro coletou dados de 24 hospitais e maternidades brasileiras entre março e julho de 2020. O estudo encontrou que, nas salas de parto, 98,5\% dos serviços proibiam o contato pele a pele imediato e ininterrupto entre as mães e seus bebês e não apoiavam as mães para iniciar a amamentação na primeira hora. No alojamento conjunto, $98,5 \%$ dos serviços permitiam a amamentação ao mesmo tempo em que implantavam práticas de higiene respiratória para prevenir a transmissão do COVID-19. Acompanhantes eram proibidos em 83,3\% dos hospitais. A extração mamária hospitalar e domiciliar foi permitida $(87,5 \%)$, mas a doação de leite materno não foi aceita $(95,8 \%)$. Faltaram ainda orientações quanto ao uso de estratégias de conforto infantil. Desta forma, a presente pesquisa evidencia qu,e no Brasil, os hospitais não têm seguido as recomendações para proteger, promover e apoiar a amamentação durante a pandemia de COVID-19. O desacordo entre as diretrizes internacionais tem sido um grande problema. A ausência de recomendações sobre o apoio ao aleitamento materno durante a pandemia gerou dificuldades no desenvolvimento de padrões entre hospitais de diferentes regiões do Brasil e de outros países do mundo. Este estudo nos mostra a necessidade de a comunidade científica discutir como melhorar os serviços de cuidados materno-infantis para proteger, com a devida segurança, a amamentação na atual pandemia.

Esta revisão apresenta limitações por retratar uma doença recém-descoberta e ainda em dinâmico estudo o que pode corroborar um número ainda incipiente de estudos primários de elevada qualidade metodológica.

Assim, ressalta-se que uma avaliação cuidadosa dos benefícios da amamentação no contexto da COVID-19 e das ações baseadas em evidências são de notável utilidade para a população nestes tempos (Ranganathan et al., 2020). Com base nos dados descritos, enfatiza-se a relevância deste estudo, no sentido de trazer resultados que corroboram o incentivo e a promoção do aleitamento materno no contexto da COVID-19.

\section{Conclusão}

Nos estudos analisados nesta revisão de escopo, os principais achados apontam que as taxas de transmissão de COVID-19 da mãe para o lactente parecem não ser aumentadas devido ao aleitamento materno, desde que respeitadas as devidas medidas sanitárias descritas. Conclui-se, pois, que a amamentação com seus inúmeros benefícios, mesmo no contexto da infecção pelo SARS-CoV-2, deve ser promovida e que novos estudos são necessários para estabelecer, com significativa evidência científica, a segurança da amamentação no contexto da pandemia pela COVID-19. 
Com a atual revisão sugere-se que novos estudos, com dados primários e de acompanhamento, sejam realizados para esclarecimento do impacto da pandemia de COVID-19 nas prevalências de aleitamento materno, bem como da segurança quanto à doação de leite a bancos de leite humano.

\section{Referências}

Bertino, E., Moro, G. E., De Renzi, G., Viberti, G., Cavallo, R., Coscia, A., Rubino, C., Tonetto, P., Sottemano, S., Campagnoli, M. F., Soldi, A., Mostert, M., Cresi, F., Lembo, D., \& Milk, C. R. G. on S.-C.-2 in H. (2020). Detection of SARS-CoV-2 in Milk From COVID-19 Positive Mothers and Follow-Up of Their Infants. Frontiers in Pediatrics, 8, 597699

Cabral, I. E., Pestana-Santos, M., Ciuffo, L. L., Nunes, Y. do R., \& Lomba, M. de L. L. de F. (2021). Child health vulnerabilities during the COVID-19 pandemic in Brazil and Portugal. Revista Latino-Americana de Enfermagem, 29, e3422.

Cabrera-Rubio, R., Kunz, C., Rudloff, S., García-Mantrana, I., Crehuá-Gaudiza, E., Martínez-Costa, C., \& Carmen Collado, M. (2019). Association of Maternal Secretor Status and Human Milk Oligosaccharides with Milk Microbiota: An Observational Pilot Study. Journal of Pediatric Gastroenterology and Nutrition, 68(2), 256-263.

Carmo, G. do, \& Portugual, P. (2020). Os diversos benefícios do aleitamento materno (Trabalho de Conclusão de Curso). Centro Universitário de Brasília, Brasília, DF, Brasil.

Ceulemans, M., Verbakel, J. Y., Calsteren, K. Van, Eerdekens, A., Allegaert, K., \& Foulon, V. (2020). SARS-CoV-2 Infections and Impact of the COVID-19 Pandemic in Pregnancy and Breastfeeding: Results from an Observational Study in Primary Care in Belgium. International Journal of Environmental Research and Public Health 2020, 17(18), 6766.

Chen, H., Guo, J., Wang, C., Luo, F., Yu, X., Zhang, W., Li, J., Zhao, D., Xu, D., Gong, Q., Liao, J., Yang, H., Hou, W., \& Zhang, Y. (2020). Clinical characteristics and intrauterine vertical transmission potential of COVID-19 infection in nine pregnant women: a retrospective review of medical records. The Lancet, 395(10226), 809-815

Davanzo, R., Moro, G., Sandri, F., Agosti, M., Moretti, C., \& Mosca, F. (2020). Breastfeeding and coronavirus disease-2019: Ad interim indications of the Italian Society of Neonatology endorsed by the Union of European Neonatal \& Perinatal Societies. Maternal \& Child Nutrition, 16(3), e13010.

De Rose, D. U., Piersigilli, F., Ronchetti, M. P., Santisi, A., Bersani, I., Dotta, A., Danhaive, O., \& Auriti, C. (2020). Novel Coronavirus disease (COVID-19) in newborns and infants: what we know so far. Italian Journal of Pediatrics, 46(1), 1-8.

Dimopoulou, D., Triantafyllidou, P., Daskalaki, A., Syridou, G., \& Papaevangelou, V. (2020). Breastfeeding during the novel coronavirus (COVID-19) pandemic: guidelines and challenges. Journal of Maternal-Fetal and Neonatal Medicine, 8, 1-7.

Fernández-Carrasco, F., Vázquez-Lara, J., González-Mey, U., Gómez-Salgado, J., Parron-Carreño, T., \& Rodríguez-Díaz, L. (2020). Infección por coronavirus Covid-19 y lactancia materna: una revisión exploratoria. Revista Española de Salud Pública, 94(1), 1-9.

Genoni, G., Conio, A., Binotti, M., Manzoni, P., Castagno, M., Rabbone, I., \& Monzani, A. (2020). Management and Nutrition of Neonates during the COVID-19 Pandemic: A Review of the Existing Guidelines and Recommendations. American Journal of Perinatology, 37(2), 46-53.

Gomes, R. P., Silva, P. C. V. da, Silva, C. E. A. L. da, Santos, J. C. A., Macário, F. N., Ramos, F. M. F., Sousa, J. I. B. de, \& Silva, R. R. V. da. (2020). Fatores que levam ao desmame precoce do aleitamento materno / Factors that take early mother's breastfeeding. Brazilian Journal of Development, 6(12), $100688-100700$.

Gonçalves-Ferri, W. A., Pereira-Cellini, F. M., Coca, K., Aragon, D. C., Nader, P., Lyra, J. C., do Vale, M. S., Marba, S., Araujo, K., Dias, L. A., de Lima Mota Ferreira, D. M., Nieto, G., Anchieta, L. M., de Cássia Silveira, R., de Moura, M. D. R., Tuma Calil, V. M. L., Moraes, V. C. C., de Almeida, J. H. C. L., Magalhães, M., ... de Oliveira Pinto, R. M. (2021). The impact of coronavirus outbreak on breastfeeding guidelines among Brazilian hospitals and maternity services: a cross-sectional study. International Breastfeeding Journal, 16(1), 1-11.

Johns Hopkins. (2021). COVID-19 Dashboard by the Center for Systems Science and Engineering at Johns Hopkins University. Maryland: Johns Hopkins.

Kuehn, B. M. (2021). COVID-19 Precautions Hamper Breastfeeding Support. JAMA, 325(2), 122-122.

Ministério da Saúde. Secretaria de Atenção Primária. Departamento de Promoção à Saúde. (2019). Guia alimentar para crianças brasileiras menores de dois anos. Brasília: Ministério da Saúde.

Ministério da Saúde, Secretaria de Atenção Primária à Saúde. (2020). Protocolo de Manejo Clínico do Coronavírus (COVID-19) na Atenção Primária à Saúde. Brasília: Ministério da Saúde.

Ministério da Saúde. (2021). Boletim Epidemiológico Especial: Doença pelo Novo Coronavírus - COVID-19. Brasília: Ministério da Saúde.

Ng, Y. P. M., Low, Y. F., Goh, X. L., Fok, D., \& Amin, Z. (2020). Breastfeeding in COVID-19: A Pragmatic Approach. American Journal of Perinatology, 37, 1377-1384.

Nuriel-Ohayon, M., Neuman, H., Ziv, O., Belogolovski, A., Barsheshet, Y., Bloch, N., Uzan, A., Lahav, R., Peretz, A., Frishman, S., Hod, M., Hadar, E., Louzoun, Y., Avni, O., \& Koren, O. (2019). Progesterone Increases Bifidobacterium Relative Abundance during Late Pregnancy. Cell Reports, 27(3), 730736.

Organização Pan-Americana da Saúde. (2020). Aleitamento materno e a doença causada pelo novo coronavírus (COVID-19). Informações científicas: 23 de 
Research, Society and Development, v. 10, n. 12, e237101220215, 2021 (CC BY 4.0) | ISSN 2525-3409 | DOI: http://dx.doi.org/10.33448/rsd-v10i12.20215

junho de 2020. Brasília: Organização Pan-Americana da Saúde.

Patil, U. P., Maru, S., Krishnan, P., Carroll-Bennett, R., Sanchez, J., Noble, L., \& Wasserman, R. (2020). Newborns of COVID-19 mothers: short-term outcomes of colocating and breastfeeding from the pandemic's epicenter. Journal of Perinatology, 40(10), 4.

Paz, M. M. S. da, Almeida, M. de O., Cabral, N. O., Assis, T. J. C. F. de, \& Mendes, C. K. T. T. (2021). Barriers imposed in the relationship between puerperal mothers and newborns in the pandemic scenario of COVID-19. Revista Brasileira de Saude Materno Infantil, 21(supl.1), 229-232.

Pereira, A., Cruz-Melguizo, S., Adrien, M., Fuentes, L., Marin, E., Forti, A., \& Perez-Medina, T. (2020). Breastfeeding mothers with COVID-19 infection: a case series. International Breastfeeding Journal, 15, 69.

Ranganathan, R., Khan, A. M., \& Chhabra, P. (2020). Antenatal care, care at birth, and breastfeeding during the Coronavirus (COVID-19) pandemic. Indian Journal of Community Health, 32(1), 15-18.

Rimes, K. A., Oliveira, M. I. C. de, \& Boccolini, C. S. (2019). Maternity leave and exclusive breastfeeding. Revista de Saúde Pública, 53, 10.

Salvatore, C. M., Han, J.-Y., Acker, K. P., Tiwari, P., Jin, J., Brandler, M., Cangemi, C., Gordon, L., Parow, A., DiPace, J., \& DeLaMora, P. (2020). Neonatal management and outcomes during the COVID-19 pandemic: an observation cohort study. The Lancet. Child \& Adolescent Health, 4(10), 727.

Santiano, F. E., Arboccó, F. C. V., Bruna, F. A., Zyla, L. E., Sasso, C. V., Gómez, S., Pistone-Creydt, V., López-Fontana, C. M., \& Carón, R. W. (2021). The epigenetic role of breastfeeding in mammary differentiation. Journal of Developmental Origins of Health and Disease, 12(4), 578-586.

Sociedade Brasileira de Pediatria, Departamento Científico De Aleitamento Materno. (2018). Amamentação: A base da vida. Rio de Janeiro: Sociedade Brasileira de Pediatria.

Sullivan, S. E., \& Thompson, L. A. (2020). Best Practices for COVID-19-Positive or Exposed Mothers-Breastfeeding and Pumping Milk. JAMA Pediatrics, $174(12), 1228-1228$.

Thanigainathan, S., Kaliyaperumal, V., Sivanandan, S., Rengaraj, S., Dhodapkar, R., \& Bethou, A. (2021). Is SARS-CoV-2 Transmitted Through Breastfeeding? Indian Journal of Pediatrics, 88(8), 800-801.

The Joanna Briggs Institute. (2014). Joanna Briggs Institute Reviewers Manual: 2014 edition. Adelaide: The Joanna Briggs Institute.

The Joanna Briggs Institute. (2013). JBI Grades of Recommendation. Adelaide: The Joanna Briggs Institute.

Tricco, A. C., Lillie, E., Zarin, W., O’Brien, K. K., Colquhoun, H., Levac, D., Moher, D., Peters, M. D. J., Horsley, T., Weeks, L., Hempel, S., Akl, E. A., Chang, C., McGowan, J., Stewart, L., Hartling, L., Aldcroft, A., Wilson, M. G., Garritty, C., ... Straus, S. E. (2018). PRISMA extension for scoping reviews (PRISMA-ScR): Checklist and explanation. Annals of Internal Medicine, 169(7), 467-473.

Vassilopoulou, E., Feketea, G., Koumbi, L., Mesiari, C., Berghea, E. C., \& Konstantinou, G. N. (2021). Breastfeeding and COVID-19: From Nutrition to Immunity. Frontiers in Immunology, 12, 661806.

Victora, C. G., Bahl, R., Barros, A. J. D., França, G. V. A., Horton, S., Krasevec, J., Murch, S., Sankar, M. J., Walker, N., \& Rollins, N. C. (2016). Breastfeeding in the 21st century: epidemiology, mechanisms, and lifelong effect. The Lancet, 387(10017), 475-490.

Walker, G. J., Clifford, V., Bansal, N., Stella, A. O., Turville, S., Stelzer-Braid, S., Klein, L. D., \& Rawlinson, W. (2020). SARS-CoV-2 in human milk is inactivated by Holder pasteurisation but not cold storage. Journal of Paediatrics and Child Health, 56(12), 1872-1874.

Walker, K., O’Donoghue, K., Grace, N., Dorling, J., Comeau, J., Li, W., \& Thornton, J. (2020). Maternal transmission of SARS-COV-2 to the neonate, and possible routes for such transmission: a systematic review and critical analysis. BJOG: An International Journal of Obstetrics \& Gynaecology, 127(11), 13241336.

Williams, J., Namazova-Baranova, L., Weber, M., Vural, M., Mestrovic, J., Carrasco-Sanz, A., Breda, J., Berdzuli, N., \& Pettoello-Mantovani, M. (2020). The Importance of Continuing Breastfeeding during Coronavirus Disease-2019: In Support of the World Health Organization Statement on Breastfeeding during the Pandemic. The Journal of Pediatrics, 223, 234-236.

Yang, N., Che, S., Zhang, J., Wang, X., Tang, Y., Wang, J., Huang, L., Wang, C., Zhang, H., Baskota, M., Ma, Y., Zhou, Q., Luo, X., Yang, S., Feng, X., Li, W., Fukuoka, T., Ahn, H. S., Lee, M. S., \& Group, on behalf of C.-19 E. and R. W. (2020). Breastfeeding of infants born to mothers with COVID-19: a rapid review. Annals of Translational Medicine, 8(10), 618-618. 